\title{
Non-bipartite distance-regular graphs with a small smallest eigenvalue
}

\author{
Zhi Qiao \\ School of Mathematical Sciences \\ Sichuan Normal University \\ 610068, Sichuan, PR China \\ zhiqiao@sicnu.edu.cn
}

\author{
Yifan Jing \\ Department of Mathematics \\ University of Illinois at Urbana Champaign \\ Urbana, IL, 61801, USA \\ yifanjing17@gmail.com
}

\author{
Jack Koolen* \\ School of Mathematical Sciences \\ University of Science and Technology of China \\ and \\ Wen-Tsun Wu Key Laboratory of \\ the Chinese Academy of Sciences \\ 230026, Anhui, PR China \\ koolen@ustc.edu.cn
}

Submitted: Dec 4, 2018; Accepted: May 21, 2019; Published: Jun 21, 2019

(C) The authors. Released under the CC BY license (International 4.0).

\begin{abstract}
In 2017, Qiao and Koolen showed that for any fixed integer $D \geqslant 3$, there are only finitely many non-bipartite distance-regular graphs with $\theta_{\min } \leqslant-\alpha k$, where $0<\alpha<1$ is any fixed number. In this paper, we will study non-bipartite distanceregular graphs with relatively small $\theta_{\min }$ compared with $k$. In particular, we will show that if $\theta_{\min }$ is relatively close to $-k$, then the odd girth $g$ must be large. Also we will classify the non-bipartite distance-regular graphs with $\theta_{\min } \leqslant-\frac{D-1}{D} k$ for $D=4,5$.
\end{abstract}

Mathematics Subject Classifications: 05C75, 05E30, 05C50

Key words: Distance-regular graphs, Smallest eigenvalue, Odd girth AMS classification: 05C75, 05E30, 05C50

\footnotetext{
*Corresponding author
} 


\section{Introduction}

The odd girth of a non-bipartite graph is the length of its shortest odd cycle. Let $\Gamma$ be a non-bipartite distance-regular graph with valency $k$, diameter $D$, odd girth $g$ and smallest eigenvalue $\theta_{\text {min }}$. In [6], Qiao and Koolen showed that for any fixed integer $D \geqslant 3$, there are only finitely many such graphs with $\theta_{\min } \leqslant-\alpha k$, where $0<\alpha<1$ is any fixed number. In this paper, we will study non-bipartite distance-regular graphs with relatively small $\theta_{\min }$ compared with $k$. In the next result, we will show that if $\theta_{\min }$ is relatively close to $-k$, then the odd girth $g$ must be large.

Theorem 1. Let $\Gamma$ be a non-bipartite distance-regular graph with valency $k$ and odd girth $g$, having smallest eigenvalue $\theta_{\min }$. Then there exists a constant $\varepsilon(g)>0$ such that $\theta_{\min } \geqslant-(1-\varepsilon(g)) k$.

Remark 2. The positive constant $\varepsilon(g)$ goes to 0 as the odd girth $g$ goes to $\infty$. For example, the $(2 t+1)$-gon has valency $k=2$, odd girth $g=2 t+1$ and smallest eigenvalue $\theta_{\min }=2 \cos \left(\frac{2 t \pi}{2 t+1}\right)$. Thus, $\varepsilon(g) \leqslant 1+\frac{\theta_{\min }}{k}=2 \cos ^{2}\left(\frac{t \pi}{2 t+1}\right)$.

In [6], Qiao and Koolen classified non-bipartite distance-regular graphs with valency $k$, diameter $D \leqslant 3$ and smallest eigenvalue $\theta_{\min } \leqslant-k / 2$. Using Theorem 1 , we will classify non-bipartite distance-regular graphs with valency $k$, diameter $D$ and smallest eigenvalue $\theta_{\min } \leqslant-\frac{D-1}{D} k$, when $D=4$ or 5 .

Theorem 3. Let $\Gamma$ be a non-bipartite distance-regular graph with valency $k$, diameter $D$ and smallest eigenvalue $\theta_{\min } \leqslant-\frac{D-1}{D} k$.

i) If $D=4$, then $\Gamma$ is one of the following graph

a) the Coxeter graph with intersection array $\{3,2,2,1 ; 1,1,1,2\}$,

b) the 9-gon with intersection array $\{2,1,1,1 ; 1,1,1,1\}$,

c) the Odd graph $O_{5}$ with intersection array $\{5,4,4,3 ; 1,1,2,2\}$,

d) the folded 9-cube with intersection array $\{9,8,7,6 ; 1,2,3,4\}$.

ii) If $D=5$, then $\Gamma$ is one of the following graph

a) the 11-gon with intersection array $\{2,1,1,1,1 ; 1,1,1,1,1\}$,

b) the Odd graph $O_{6}$ with intersection array $\{6,5,5,4,4 ; 1,1,2,2,3\}$,

c) the folded 11-cube with intersection array $\{11,10,9,8,7 ; 1,2,3,4,5\}$.

This paper is organized as follows. In the next section, we give the definitions and some preliminary results. In Section 3, we give a proof of Theorem 1. In the last section, we give a proof of Theorem 3 . 


\section{Preliminaries}

For more background, see [4] and [7].

All the graphs considered in this paper are finite, undirected and simple. Let $\Gamma$ be a graph with vertex set $V=V(\Gamma)$ and edge set $E=E(\Gamma)$. Denote $x \sim y$ if the vertices $x, y \in V$ are adjacent. The distance $d(x, y)=d_{\Gamma}(x, y)$ between two vertices $x, y \in V(\Gamma)$ is the length of a shortest path connecting $x$ and $y$. The maximum distance between two vertices in $\Gamma$ is the diameter $D=D(\Gamma)$. We use $\Gamma_{i}(x)$ for the set of vertices at distance $i$ from $x$ and write, for the sake of simplicity, $\Gamma(x):=\Gamma_{1}(x)$. The degree of $x$ is the number $|\Gamma(x)|$ of vertices adjacent to it. A graph is regular with valency $k$ if the degree of each of its vertices is $k$. The girth and odd girth of a graph is the length of its shortest cycle, and shortest odd cycle, respectively. A graph $\Gamma$ is called bipartite if it has no odd cycle.

A connected graph $\Gamma$ with diameter $D$ is called distance-regular if there are integers $b_{i}, c_{i}(i=0,1, \ldots, D)$ such that for any two vertices $x, y \in V(\Gamma)$ with $d(x, y)=i$, there are exactly $c_{i}$ neighbors of $y$ in $\Gamma_{i-1}(x)$ and $b_{i}$ neighbors of $y$ in $\Gamma_{i+1}(x)$, where we define $b_{D}=c_{0}=0$. In particular, $\Gamma$ is a regular graph with valency $k:=b_{0}$. We define $a_{i}:=k-b_{i}-c_{i}(i=0,1, \ldots, D)$ for notational convenience. Note that $a_{i}=\left|\Gamma(y) \cap \Gamma_{i}(x)\right|$ holds for any two vertices $x, y$ with $d(x, y)=i(i=0,1, \ldots, D)$.

For a distance-regular graph $\Gamma$ and a vertex $x \in V(\Gamma)$, we denote $k_{i}:=\left|\Gamma_{i}(x)\right|$ and $p_{i j}^{h}:=\left|\left\{w \mid w \in \Gamma_{i}(x) \cap \Gamma_{j}(y)\right\}\right|$ for any $y \in \Gamma_{h}(x)$. It is easy to see that $k_{i}=$ $b_{0} b_{1} \cdots b_{i-1} /\left(c_{1} c_{2} \cdots c_{i}\right)$ and hence it does not depend on $x$. The numbers $a_{i}, b_{i}$ and $c_{i}(i=$ $0,1, \ldots, D)$ are called the intersection numbers, and the array $\left\{b_{0}, b_{1}, \ldots, b_{D-1} ; c_{1}, c_{2}, \ldots\right.$, $\left.c_{D}\right\}$ is called the intersection array of $\Gamma$. The matrix $L$ is called the intersection matrix of $\Gamma$, where

$$
L=\left(\begin{array}{cccccc}
a_{0} & b_{0} & 0 & & & \\
c_{1} & a_{1} & b_{1} & & 0 & \\
& c_{2} & a_{2} & \cdot & & \\
& & \cdot & \cdot & \cdot & \\
& 0 & & \cdot & \cdot & b_{D-1} \\
& & & & c_{D} & a_{D}
\end{array}\right) .
$$

Let $\Gamma$ be a distance-regular graph with $v$ vertices and diameter $D$. Let $A_{i}(i=$ $0,1, \ldots, D)$ be the $(0,1)$-matrix whose rows and columns are indexed by the vertices of $\Gamma$ and the $(x, y)$-entry is 1 whenever $d(x, y)=i$ and 0 otherwise. We call $A_{i}$ the distance- $i$ matrix and $A:=A_{1}$ the adjacency matrix of $\Gamma$. The eigenvalues $\theta_{0}>\theta_{1}>\cdots>\theta_{D}$ of the graph $\Gamma$ are just the eigenvalues of its adjacency matrix $A$. We denote $m_{i}$ the multiplicity of $\theta_{i}$. Note that the $D+1$ distinct eigenvalues of $\Gamma$ are precisely the eigenvalues of $L$ (see [7, Proposition 2.7]).

For each eigenvalue $\theta_{i}$ of $\Gamma$, let $U_{i}$ be a matrix with its columns forming an orthonormal basis for the eigenspace associated with $\theta_{i}$. And $E_{i}:=U_{i} U_{i}^{T}$ is called the minimal idempotent associated with $\theta_{i}$, satisfying $E_{i} E_{j}=\delta_{i j} E_{j}$ and $A E_{i}=\theta_{i} E_{i}$, where $\delta_{i j}$ is the Kronecker delta. Note that $v E_{0}$ is the all-ones matrix $J$.

The set of distance matrices $\left\{A_{0}=I, A_{1}, A_{2}, \ldots, A_{D}\right\}$ forms a basis of a commutative $\mathbb{R}$-algebra $\mathcal{A}$, known as the Bose-Mesner algebra. The set of minimal idempotents $\left\{E_{0}=\right.$ 
$\left.\frac{1}{v} J, E_{1}, E_{2}, \ldots, E_{D}\right\}$ is another basis of $\mathcal{A}$. There exist $(D+1) \times(D+1)$ matrices $P$ and $Q$ (see $[4, \mathrm{p} .45]$ ), such that the following relations hold

$$
A_{i}=\sum_{j=0}^{D} P_{j i} E_{j} \quad \text { and } \quad E_{i}=\frac{1}{v} \sum_{j=0}^{D} Q_{j i} A_{j} \quad(i=0,1, \ldots, D) .
$$

Note that $Q_{0 i}=m_{i}$ (see [4, Lemma 2.2.1]).

Let $E_{i}=U_{i} U_{i}^{T}$ be the minimal idempotent associated with $\theta_{i}$, where the columns of $U_{i}$ form an orthonormal basis of the eigenspace associated with $\theta_{i}$. We denote the $x$-th row of $\sqrt{v / m_{i}} U_{i}$ by $\hat{x}$. Note that $E_{i} \circ A_{j}=\frac{1}{v} Q_{j i} A_{j}$, hence all the vectors $\hat{x}$ are unit vectors and the cosine of the angle between two vectors $\hat{x}$ and $\hat{y}$ is $u_{j}\left(\theta_{i}\right):=\frac{Q_{j i}}{Q_{0 i}}$, where $d(x, y)=j$. The map $x \mapsto \hat{x}$ is called a normalized representation and the sequence $\left(u_{j}\left(\theta_{i}\right)\right)_{j=0}^{D}$ is called the standard sequence of $\Gamma$, associated with $\theta_{i}$. As $A U_{i}=\theta_{i} U_{i}$, we have $\theta_{i} \hat{x}=\sum_{y \sim x} \hat{y}$, and hence the following holds:

$$
\left\{\begin{array}{l}
c_{j} u_{j-1}\left(\theta_{i}\right)+a_{j} u_{j}\left(\theta_{i}\right)+b_{j} u_{j+1}\left(\theta_{i}\right)=\theta_{i} u_{j}\left(\theta_{i}\right) \quad(j=1,2, \ldots, D-1), \\
c_{D} u_{D-1}\left(\theta_{i}\right)+a_{D} u_{D}\left(\theta_{i}\right)=\theta_{i} u_{D}\left(\theta_{i}\right),
\end{array}\right.
$$

with $u_{0}\left(\theta_{i}\right)=1$ and $u_{1}\left(\theta_{i}\right)=\frac{\theta_{i}}{k}$.

Lemma 4. (c.f. [7, Theorem 2.8]) Let $\Gamma$ be a distance-regular graph with diameter $D$ and $v$ vertices. Let $\theta$ be an eigenvalue of $\Gamma$ and $\left(u_{i}\right)_{i=0}^{D}$ be the standard sequence associated with $\theta$. Then the multiplicity $m(\theta)$ of $\theta$ as an eigenvalue of $\Gamma$ satisfies

$$
\begin{aligned}
m(\theta) & =\frac{v}{\sum_{i=0}^{D} k_{i} u_{i}^{2}}, \\
& \leqslant \max \left\{\frac{1}{u_{1}^{2}}, \ldots, \frac{1}{u_{j-1}^{2}}, \frac{\sum_{i=j}^{D} k_{i}}{k_{j} u_{j}^{2}}\right\} \quad(j=1,2, \ldots, D) .
\end{aligned}
$$

Proof. Equation (3) follows from [7, Theorem 2.8]. We only give a proof of Equation (4).

$$
\begin{aligned}
& \frac{v}{\sum_{i=0}^{D} k_{i} u_{i}^{2}}=\frac{\sum_{i=0}^{D} k_{i}}{\sum_{i=0}^{D} k_{i} u_{i}^{2}} \leqslant \max \left\{\frac{\sum_{i=0}^{j-1} k_{i}}{\sum_{i=0}^{j-1} k_{i} u_{i}^{2}}, \frac{\sum_{i=j}^{D} k_{i}}{\sum_{i=j}^{D} k_{i} u_{i}^{2}}\right\}, \\
& \frac{\sum_{i=0}^{j-1} k_{i}}{\sum_{i=0}^{j-1} k_{i} u_{i}^{2}} \leqslant \max \left\{\frac{1}{u_{1}^{2}}, \ldots, \frac{1}{u_{j-1}^{2}}\right\}, \\
& \frac{\sum_{i=j}^{D} k_{i}}{\sum_{i=j}^{D} k_{i} u_{i}^{2}} \leqslant \frac{\sum_{i=j}^{D} k_{i}}{k_{j} u_{j}^{2}} .
\end{aligned}
$$


Lemma 5. ([4, Proposition 4.1.6]) Let $\Gamma$ be a distance-regular graph with valency $k$ and diameter $D$. Then the following conditions hold

i) $1=c_{1} \leqslant c_{2} \leqslant \cdots \leqslant c_{D}$,

ii) $k=b_{0} \geqslant b_{1} \geqslant \cdots \geqslant b_{D-1}$,

iii) $k_{i}$ 's $(i=1,2, \ldots, D)$ are positive integers,

iv) the multiplicities are positive integers.

Lemma 6. (c.f. [2, Proposition 3.1]) Let $\Gamma$ be a non-bipartite distance-regular graph with valency $k$ and odd girth $g=2 t+1$. Then

$$
\sum_{i=0}^{t} p_{i}(\eta) u_{i} \geqslant 0
$$

where $\left(u_{i}\right)_{i=0}^{D}$ is the standard sequence associated with the smallest eigenvalue $\theta_{\min }, \eta$ is any eigenvalue of the $g$-gon, and $p_{i}(x)$ is defined as the following

$$
\begin{aligned}
& p_{0}(x)=1, \\
& p_{1}(x)=x \\
& p_{2}(x)=x^{2}-2 \\
& p_{i}(x)=x p_{i-1}(x)-p_{i-2}(x) \quad(i=3,4, \ldots, t) .
\end{aligned}
$$

Proof. Let $\Delta$ be any $g$-gon in $\Gamma$. Let $B_{i}(i=0,1, \ldots, t)$ be the matrix with rows and columns indexed by $V(\Delta)$, where the $(v, w)$-entry is 1 whenever $d_{\Gamma}(v, w)=i$ and 0 otherwise. Note that $d_{\Delta}(v, w)=d_{\Gamma}(v, w)$ for any two vertices $v, w \in V(\Delta)$, and we have $B_{i}=p_{i}\left(B_{1}\right)$ with $p_{i}(x)$ as Equation (6). By [2, Proposition 3.1], for any eigenvalue $\eta$ of $\Delta$, we have Equation (5).

Lemma 7. (c.f. [6, Lemma 5.2]) Let $\Gamma$ be a distance-regular graph with valency $k$ and smallest eigenvalue $\theta_{\min }$. If $a_{1}=0$ and $\theta_{\min }<\frac{12-5 k}{7}$, then $c_{2} \leqslant 2$.

Proof. Choose two vertices $x, y \in V(\Gamma)$ with $d(x, y)=2$. As $a_{1}=0$, the subgraph induced on $\{x, y\} \cup(\Gamma(x) \cap \Gamma(y))$ is a $K_{2, c_{2}}$. Let $x \mapsto \hat{x}$ be a normalized representation associated with $\theta=\theta_{\min }$. Consider the Gram matrix of the image of the $K_{2, c_{2}}$ with the bipartition, we see that

$$
Q=\left(\begin{array}{cc}
\frac{1}{2}\left(1+u_{2}\right) & u_{1} \\
u_{1} & \frac{1}{c_{2}}\left(1+\left(c_{2}-1\right) u_{2}\right)
\end{array}\right)
$$

is positive semidefinite, by [4, Proposition 3.7.1 (iii)]. Then $(1,1) Q(1,1)^{t} \geqslant 0$, which in turn implies $\left(u_{1}+u_{2}\right)\left(\left(2+c_{2}\right) \frac{1-u_{2}}{u_{1}+u_{2}}+4 c_{2}\right) \geqslant 0$. As $a_{1}=0$, we see $u_{1}+u_{2}=\frac{(\theta+k)(\theta-1)}{k(k-1)}<0$, that is $\frac{4 c_{2}}{2+c_{2}} \leqslant-\frac{1-u_{2}}{u_{1}+u_{2}}=\frac{k-\theta}{1-\theta}$. When $k>1$, we have $\theta<\frac{12-5 k}{7}<\frac{4-k}{3}$ and $c_{2} \leqslant \frac{2 k-2 \theta}{4-3 \theta-k}<$ 3 . 
Lemma 8. Let $\Gamma$ be a distance-regular graph with valency $k$ and diameter $D$, having smallest eigenvalue $\theta_{\min }$ with associated standard sequence $\left(u_{i}\right)_{i=0}^{D}$. Then

$$
\left|u_{i+1}\right| \geqslant \frac{\left|\left(\theta_{\min }-a_{i}\right) u_{i}\right|-c_{i}\left|u_{i-1}\right|}{b_{i}} \quad(i=1, \ldots, D-1) .
$$

Proof. By Equation (2) we see that $u_{i+1}=\frac{\left(\theta_{\min }-a_{i}\right) u_{i}-c_{i} u_{i-1}}{b_{i}}(i=1, \ldots, D-1)$. As $\theta_{\min }<0$ is the smallest eigenvalue, by [4, Corollary 4.1.2], we see that $u_{i+1},-u_{i}$ and $u_{i-1}$ has the same sign. The result follows.

\section{Main Theorem}

In this section we will prove our main result.

Proof of Theorem 1. If $g=3$, then $\theta_{\min } \geqslant-\frac{k}{2}$ by [7, Proposition 2.11]. So we may assume $g \geqslant 5$. Let $t=\frac{g-1}{2}$ and $\Delta$ be a $g$-gon in $\Gamma$. Let $\left(u_{i}\right)_{i=0}^{D}$ be the standard sequence associated with the smallest eigenvalue $\theta=\theta_{\min }$.

Assume $c_{t} \leqslant \zeta k$ for some $\zeta \leqslant \frac{1}{2}$. By Lemma 6 , we have $\sum_{i=0}^{t} p_{i}(\eta) u_{i} \geqslant 0$, where $p_{i}(x)$ is as Equation (6).

We claim that there exist constants $N_{i}$ such that

$$
\left|u_{i}-\left(\frac{\theta}{k}\right)^{i}\right| \leqslant N_{i} \zeta \quad(i=0,1, \ldots, t),
$$

with

$$
N_{i}= \begin{cases}0, & i=0,1, \\ 2 N_{i-1}+4, & i=2,3 \ldots, t .\end{cases}
$$

Note that $\left|u_{0}-1\right|=\left|u_{1}-\frac{\theta}{k}\right|=0$. Assume $\left|u_{i}-\left(\frac{\theta}{k}\right)^{i}\right| \leqslant N_{i} \zeta$ for some $1 \leqslant i \leqslant t-1$. As $c_{t} \leqslant \zeta k$, we see that $c_{i} \leqslant c_{t} \leqslant \zeta k$ and $b_{i}=k-c_{i} \geqslant(1-\zeta) k$. Then

$$
\begin{aligned}
\left|u_{i+1}-\left(\frac{\theta}{k}\right)^{i+1}\right| & =\left|\frac{\theta u_{i}-c_{i} u_{i-1}}{b_{i}}-\left(\frac{\theta}{k}\right)^{i+1}\right| \\
& \leqslant\left|\frac{\theta}{b_{i}} u_{i}-\frac{\theta}{b_{i}}\left(\frac{\theta}{k}\right)^{i}\right|+\left|\frac{\theta}{b_{i}}\left(\frac{\theta}{k}\right)^{i}-\left(\frac{\theta}{k}\right)^{i+1}\right|+\frac{c_{i}}{b_{i}} \cdot\left|u_{i-1}\right| \\
& =\left|\frac{\theta}{b_{i}}\right| \cdot\left|u_{i}-\left(\frac{\theta}{k}\right)^{i}\right|+\frac{c_{i}}{b_{i}} \cdot\left|\left(\frac{\theta}{k}\right)^{i+1}\right|+\frac{c_{i}}{b_{i}} \cdot\left|u_{i-1}\right| \\
& \leqslant \frac{k}{b_{i}} \cdot N_{i} \zeta+\frac{c_{i}}{b_{i}}+\frac{c_{i}}{b_{i}} \\
& \leqslant N_{i+1} \zeta,
\end{aligned}
$$

where $\frac{k}{b_{i}} \leqslant \frac{1}{1-\zeta} \leqslant 2$ and $\frac{c_{i}}{b_{i}} \leqslant \frac{\zeta}{1-\zeta} \leqslant 2 \zeta\left(\zeta \leqslant \frac{1}{2}\right)$. 
Note that $p_{i}(\eta)$ is an eigenvalue of the distance- $i$ graph of $\Delta$. Hence $\left|p_{i}(\eta)\right| \leqslant 2$ $(i=0,1, \ldots, t)$, and by Equation (8), we have

$$
\begin{aligned}
\sum_{i=0}^{t} p_{i}(\eta) u_{i} & \leqslant \sum_{i=0}^{t} p_{i}(\eta)\left(\frac{\theta}{k}\right)^{i}+\sum_{i=0}^{t}\left|p_{i}(\eta)\right| \cdot\left|u_{i}-\left(\frac{\theta}{k}\right)^{i}\right| \\
& \leqslant \sum_{i=0}^{t} p_{i}(\eta)\left(\frac{\theta}{k}\right)^{i}+M_{1} \zeta
\end{aligned}
$$

where $M_{1}=\sum_{i=0}^{t} 2 N_{i}$.

By Equation (6), we see that $p_{i}(x)=\lambda_{1}^{i}+\lambda_{2}^{i}(i=1,2, \ldots, t)$, with $\lambda_{i}=\frac{1}{2}\left(x \pm \sqrt{x^{2}-4}\right)$ $(i=1,2)$. Define $f(x, y)=\sum_{i=0}^{t} p_{i}(x) y^{i}=\frac{1-\left(\lambda_{1} y\right)^{t+1}}{1-\lambda_{1} y}+\frac{1-\left(\lambda_{2} y\right)^{t+1}}{1-\lambda_{2} y}-1$. Note that the eigenvalues of $\Delta$ are $2 \cos \frac{2 \pi j}{g}(i=0,1, \ldots, g-1)$. Take $\eta=2 \cos \frac{2 \pi(t-1)}{g}$, then we see

$$
f(\eta,-1)=-M_{2},
$$

where $M_{2}=1 / \cos \frac{(t-1) \pi}{g}$. In fact,

$$
\begin{aligned}
f\left(2 \cos \frac{2 \pi j}{g},-1\right) & =\frac{1-\left(-e^{2 \pi \mathrm{i} \cdot \frac{j}{g}}\right)^{t+1}}{1-\left(-e^{2 \pi \mathrm{i} \cdot \frac{j}{g}}\right)}+\frac{1-\left(-e^{-2 \pi \mathrm{i} \cdot \frac{j}{g}}\right)^{t+1}}{1-\left(-e^{-2 \pi \mathrm{i} \cdot \frac{j}{g}}\right)}-1 \\
& =(-1)^{t} \cdot \frac{e^{2 \pi \mathrm{i} \cdot \frac{j(t+1)}{g}}+e^{-2 \pi \mathrm{i} \cdot \frac{j t}{g}}}{1+e^{2 \pi \mathrm{i} \cdot \frac{j}{g}}} \\
& =(-1)^{t+j} / \cos \frac{j \pi}{g} .
\end{aligned}
$$

Take $\zeta=\min \left\{\frac{M_{2}}{2 M_{1}}, \frac{1}{2}\right\}$. Note that $M_{1}, M_{2}$, and hence $\zeta$ is determined by $g$. By Equation (10), we see $f(\eta,-1)+M_{1} \zeta \leqslant-\frac{M_{2}}{2}<0$. We also have $f(\eta, 0)+M_{1} \zeta=$ $1+M_{1} \zeta>0$. By Equation (5) and (9), we have $0 \leqslant f\left(\eta, \frac{\theta}{k}\right)+M_{1} \zeta$. Take $-\left(1-\varepsilon_{1}(\zeta)\right)$ as the smallest root $y$ of the equation $f(\eta, y)+M_{1} \zeta=0$ in the interval $(-1,0)$. It follows that $\theta \geqslant-\left(1-\varepsilon_{1}(\zeta)\right) k$.

Now we consider the case $c_{t}>\zeta k$.

If $c_{t}>1$, then we claim that the diameter $D \leqslant \frac{4 t}{\zeta^{2}}$ and $\theta_{\min } \geqslant-\left(1-\varepsilon_{2}(\zeta)\right) k$ for some constant $\varepsilon_{2}(\zeta)>0$. Without loss of generality, we may assume $4^{i} t \leqslant D \leqslant 4^{i+1} t$ for some integer $i \geqslant 1$. If $c_{2 t-1+j}=c_{2 t-1} \neq 1$, by [7, Theorem 7.1], we see $j \leqslant 2 t-1$, that is $c_{4 t-1}>c_{2 t-1}$. Then $c_{4 t-1}=c_{2 t+j}>c_{2 t-1+j}$ for some $0 \leqslant j \leqslant 2 t-1$, and $c_{4 t-1} \geqslant 2 c_{t}$ by [7, Proposition 7.2]. This implies $k \geqslant c_{4^{i} t} \geqslant 2^{i} c_{t}$, that is $D \leqslant 4 t\left(\frac{k}{c_{t}}\right)^{2} \leqslant \frac{4 t}{\zeta^{2}}$. Then by [6, Theorem 1.1], the set $S$ of distance-regular graphs with valency $k$, diameter $D \leqslant \frac{4 t}{\zeta^{2}}$, smallest eigenvalue $\theta_{\min } \leqslant-\left(1-\varepsilon_{1}(\zeta)\right) k$ and odd girth $g$ is finite. Take

$$
\varepsilon_{2}(\zeta)= \begin{cases}\min _{\Gamma \in S} \frac{k+\theta_{\min }}{k}, & \text { if } S \neq \varnothing \\ \varepsilon_{1}(\zeta), & \text { otherwise }\end{cases}
$$


If $c_{t}=1$, then $k<\frac{1}{\zeta}$. The set $S^{\prime}$ of distance-regular graphs with valency $k<\frac{1}{\zeta}$ and odd girth $g$ is finite, by [1, Theorem 1.1]. Take

$$
\varepsilon_{3}(\zeta)= \begin{cases}\min _{\Gamma \in S^{\prime}} \frac{k+\theta_{\min }}{k}, & \text { if } S^{\prime} \neq \varnothing \\ \varepsilon_{1}(\zeta), & \text { otherwise }\end{cases}
$$

Take $\varepsilon=\min \left\{\varepsilon_{1}(\zeta), \varepsilon_{2}(\zeta), \varepsilon_{3}(\zeta)\right\}$ and the result follows.

Remark 9. When the odd girth $g=5$ and $c_{2} \leqslant \zeta k$, we may take $N_{2}=\frac{2}{1-\zeta}$. Then $f(x, y)+M_{1} \zeta=1+x y+\left(x^{2}-2\right) y^{2}+\frac{4 \zeta}{1-\zeta}$. By substituting $\eta=2 \cos \frac{2 \pi}{5}$ into $f\left(\eta, \frac{\theta}{k}\right)+M_{1} \zeta \geqslant$ 0 , we find an inequality between $\zeta$ and $\frac{\theta}{k}$. For example, if $\zeta=0.1$, then $\theta \geqslant-0.78 k$.

\section{Distance-regular graphs with relatively small $\theta_{\min }$}

In this section we study distance-regular graphs with relatively small $\theta_{\min }$. In the rest of this section we will give a proof of Theorem 3 .

Proof of Theorem 3. Assume $\Gamma$ has odd girth $g=2 t+1$. Let $\left(u_{i}\right)_{i=0}^{D}$ be the standard sequence associated with the smallest eigenvalue $\theta=\theta_{\min }$.

We first consider the case $D=4$. We may assume $k \geqslant 5$, otherwise $\Gamma$ is the 9 -gon or the Coxeter graph by [3] and [5, Theorem 1.1].

As $\theta<-\frac{k}{2}$, by [7, Proposition 2.11], we have $a_{1}=0$. If $a_{2} \neq 0$, that is $t=2$, then substitute $\eta=2 \cos \frac{2 \pi(t-1)}{g}$ into Equation (5) and we get $\frac{(k-t)(2 k+\sqrt{5} t+t+\sqrt{5}-1)}{2 k(k-1)} \geqslant 0$, which implies that $\theta \geqslant \frac{-2 k-\sqrt{5}+1}{\sqrt{5}+1}$. Combine it with $\theta \leqslant-\frac{3}{4} k$, we see that $k \leqslant 2$. Hence $a_{2}=0$. Note that $\theta \leqslant-\frac{3}{4} k<\frac{12-5 k}{7}$, by Lemma 7 , we see $c_{2} \leqslant 2$.

If $a_{3} \neq 0$, then consider

$$
\left\{\begin{array}{c}
\sum_{i=0}^{t} p_{i}(\eta) u_{i} \geqslant 0 \\
-\frac{D-1}{D} k \geqslant \theta
\end{array}\right.
$$

with $\eta=2$, we obtain that $k \leqslant 4$ if $c_{2}=1$, and $k \leqslant 8$ if $c_{2}=2$. No intersection arrays satisfy Lemma 5 , with $5 \leqslant k \leqslant 8, D=4, a_{1}=a_{2}=0 \neq a_{3}, c_{2}=2$ and $\theta_{\min } \leqslant-\frac{3}{4} k$. Hence $a_{3}=0$.

Assume $k \geqslant 36$. Since $k \geqslant 36, c_{2} \leqslant 2$ and $\theta \leqslant-\frac{3}{4} k$, by Equation (7), we obtain $\left|u_{2}\right| \geqslant 0.5500$ and $\left|u_{3}\right| \geqslant 0.3926$. Now we consider the intersection matrix $L$ of $\Gamma$, where

$$
L=\left(\begin{array}{ccccc}
0 & k & 0 & 0 & 0 \\
1 & 0 & k-1 & 0 & 0 \\
0 & c_{2} & 0 & k-c_{2} & 0 \\
0 & 0 & c_{3} & 0 & k-c_{3} \\
0 & 0 & 0 & c_{4} & k-c_{4}
\end{array}\right)
$$


We see that $k^{2}+\theta^{2} \leqslant \operatorname{tr}\left(L^{2}\right) \leqslant k^{2}+6 k+c_{4}\left(2 k-c_{4}\right)$, where $c_{2} \leqslant 2$ and $c_{3} \leqslant c_{4}$. Since $k \geqslant 36$ and $\theta \leqslant-\frac{3}{4} k$, we obtain that $\frac{c_{4}}{k} \geqslant 0.2227$. By Lemma 4 , we see that $m \leqslant \max \left\{\frac{1}{u_{1}^{2}}, \frac{1}{u_{2}^{2}}, \frac{k_{3}+k_{4}}{k_{3} u_{3}^{2}}\right\}$. Since $k_{3} b_{3}=k_{4} c_{4}$, we see $\frac{k_{3}+k_{4}}{k_{3} u_{3}^{2}} \leqslant \frac{1}{u_{3}^{2}}\left(1+\frac{k}{c_{4}}\right)$. With $\left|u_{1}\right| \geqslant \frac{3}{4}$, $\left|u_{2}\right| \geqslant 0.5500,\left|u_{3}\right| \geqslant 0.3926$ and $\frac{c_{4}}{k} \geqslant 0.2227$, we obtain $m<36$. By [4, Theorem 4.4.4], we see that $k \leqslant m<36$, a contradiction. It follows that $k \leqslant 35$. Then we check the intersection arrays satisfy Lemma 5 , with $5 \leqslant k \leqslant 35, D=4, a_{1}=a_{2}=a_{3}=0 \neq a_{4}$, $c_{2}=1$ or 2 and $\theta_{\min } \leqslant-\frac{3}{4} k$, and we get the folded 9 -cube and odd graph $O_{5}$. This shows the case $D=4$.

Now we consider the case $D=5$. Similar to the case $D=4$, we may assume $k \geqslant 5$, otherwise $\Gamma$ is the 11-gon, by [3] and [5, Theorem 1.1]. As $\theta<-\frac{k}{2}$, by [7, Proposition 2.11], we have $a_{1}=0$. Substitute $\eta=2 \cos \frac{2 \pi(t-1)}{g}$ with $t=2$ into Equation (5), we obtain $\theta \geqslant \frac{-2 k-\sqrt{5}+1}{\sqrt{5}+1}$. Together with $\theta \leqslant-\frac{4}{5} k$, we see $k \leqslant 2$, and hence $a_{2}=0$. Since $\theta \leqslant-\frac{4}{5} k<\frac{12-5 k}{7}$, by Lemma 7 , we have and $c_{2} \leqslant 2$.

If $a_{3} \neq 0$, then consider Equation (11) with $\eta=2$, we obtain that $k \leqslant 3$ if $c_{2}=1$, and $k \leqslant 5$ if $c_{2}=2$. By [4, Theorem 1.13.2], no such graphs exist with $k=5$ and $c_{2}=2$. Hence $a_{3}=0$.

We consider $a_{4} \neq 0$. If $c_{3} \leqslant 0.3750 k$, combine it with Equation (11), where $\eta=-1$ $(g=9)$, we see that $k \leqslant 24$. Assume $k \geqslant 24$, then $c_{3} \geqslant 0.3750 k$. By Equation (7), we obtain $\left|u_{2}\right| \geqslant 0.6243$ and $\left|u_{3}\right| \geqslant 0.4721$. Note $\frac{k_{4}}{k_{3}}=\frac{b_{3}}{c_{4}} \leqslant \frac{1-c_{3}}{c_{3}}$ and $\frac{k_{5}}{k_{3}}=\frac{b_{3} b_{4}}{c_{4} c_{5}} \leqslant\left(\frac{1-c_{3}}{c_{3}}\right)^{2}$. By Lemma 4, we see that

$$
m \leqslant \max \left\{\frac{1}{u_{1}^{2}}, \frac{1}{u_{2}^{2}}, \frac{1}{u_{3}^{2}}\left(1+\frac{1-c_{3}}{c_{3}}+\left(\frac{1-c_{3}}{c_{3}}\right)^{2}\right)\right\},
$$

that is $k \leqslant m \leqslant 24$ by [4, Theorem 4.4.4]. No intersection arrays satisfy Lemma 5 with $5 \leqslant k \leqslant 24, D=5, a_{1}=a_{2}=a_{3}=0 \neq a_{4}, c_{2}=1$ or 2 and $\theta \leqslant-\frac{4}{5} k$. Hence $a_{4}=0$.

Assume $k \geqslant 71$. Then by Equation (7), we see that $\left|u_{2}\right| \geqslant 0.6348$ and $\left|u_{3}\right| \geqslant 0.4994$, where $\theta_{1} \leqslant-\frac{4}{5} k, c_{2}=1$ or 2 . Then as $m \geqslant k \geqslant 71$, by Equation (12), we obtain $c_{3} \leqslant 0.2166 k$. It implies $\left|u_{4}\right| \geqslant 0.3344$ by Equation (7). Consider the intersection matrix $L$, and we see that $k^{2}+\theta^{2} \leqslant \operatorname{tr}\left(L^{2}\right) \leqslant k^{2}+6 k+4 c_{5} k-c_{5}^{2}$, which implies $c_{5} \geqslant 0.1440 k$. And we see $k \leqslant m \leqslant \min \left\{\frac{1}{u_{1}^{2}}, \frac{1}{u_{2}^{2}}, \frac{1}{u_{3}^{2}}, \frac{1}{u_{4}^{2}}\left(1+\frac{k}{c_{5}}\right)\right\} \leqslant 71$. It follows that $k \leqslant 71$. Then we check all intersection arrays satisfying Lemma 5 with $5 \leqslant k \leqslant 71, D=5, a_{1}=a_{2}=a_{3}=$ $a_{4}=0 \neq a_{5}, c_{2}=1$ or 2 and $\theta \leqslant-\frac{4}{5} k$ and we obtain the odd graph $O_{6}$ and the folded 11-cube. This shows the case $D=5$.

\section{Acknowledgments}

JHK is partially supported by the National Natural Science Foundation of China (Grant No. 11471009 and Grant No. 11671376) and by 'Anhui Initiative in Quantum Information Technologies' (Grant No. AHY150200). ZQ is partially supported by the National Natural Science Foundation of China (Grant No. 11801388). 


\section{References}

[1] S. Bang, A. Dubickas, J. Koolen, and V. Moulton. There are only finitely many distance-regular graphs of fixed valency greater than two. Adv. Math., 269:1-55, 2015.

[2] S. Bang, J. Koolen, and J. Park. Some results on the eigenvalues of distance-regular graphs. Graphs and Combin., 31:1841-1853, 2015.

[3] N. Biggs, A. Boshier, and J. Shawe-Taylor. Cubic distance-regular graphs. J. London Math. Soc. (2), 33:385-394, 1986.

[4] A. Brouwer, A. Cohen, and A. Neumaier. Distance-Regular Graphs. Springer-Verlag, 1989.

[5] A. Brouwer and J. Koolen. The distance-regular graphs of valency four. J. Algebraic Combin., 10:5-24, 1999.

[6] Z. Qiao and J. Koolen. A valency bound for distance-regular graphs. J. Combin. Theory Ser. A, 155:304-320, 2018.

[7] E. van Dam, J. Koolen, and H. Tanaka. Distance-regular graphs. Electron. J. Combin., (\#DS22), 2016. 